\title{
Dispenser printed proximity sensor on fabric for creative smart fabric applications
}

\author{
Yang Wei, Russel Torah, Yi Li and John Tudor \\ University of Southampton, \\ Southampton, United Kingdom, \\ SO17 1BJ \\ Tel: $+44(0) 2380592126$ \\ Fax: +44(0)2380592901 \\ y.wei@soton.ac.uk
}

\begin{abstract}
This paper reports, for the first time, a dispenser printed proximity sensor on fabric for wearable creative applications. The entire proximity sensor is dispenser printed onto a woven polyester/cotton fabric which is the predominant fabric used for clothing. Dispenser printing of electronic functions on fabric allows for significantly greater design freedom compared to weaving or knitting. In addition, it is a digital printing process and therefore does not require additional masks or screens compared with more traditional screen or gravure printing techniques. The printed proximity sensors are tested using an Arduino micro-controller, used throughout the smart fabrics industry, and the results show that the printed sensors detect the proximity of a human hand or limb and can therefore be used for, or integrated within, existing smart fabrics allowing interactivity.
\end{abstract}

Keywords - dispenser printing, proximity sensor, wearable electronics, smart fabric

\section{INTRODUCTION}

A proximity sensor detects the presence of an object within a specified distance. This object can be either a conductor or non-conductor depending on the sensing mechanism of the proximity sensor. Mechanisms that can be used to detect the presence of an object are: inductive [1], optical [2], ultrasonic [3] and capacitive [4]. The inductive mechanism uses an LCR oscillator in which the inductance is altered by an approaching metallic object. The detectable distance is dependent on the size of the object and the frequency of the oscillator circuit. The optical mechanism uses light reflection to detect the distance. The ultrasonic mechanism uses a similar principle but with an ultrasonic wave. The capacitive sensing mechanism alters the capacitance due to the presence of an object.

The capacitive mechanism is able to detect both conductive and non-conductive objects so is suitable for detecting approaching humans and passive objects. The capacitive mechanism also does not need a complex setup. For our purposes, it is ideally suited to a printed realisation since a simple conductive plate with any shape can be used as the sensing element. A simple sensing element of any shape is advantageous for creative applications as the sensor can be designed to any artistic shape to meet the designer's requirements.
Dispenser printing is a direct write printing technique that enables structures to be built directly from the PC design without the use of masks, allowing rapid prototyping [5]. The material is deposited through a pressurised syringe onto the substrate which is controlled in three dimensions using an XYZ stage system according to the desired printed pattern. The print resolution is dependent on the accuracy of each stage, the size of the syringe nozzle, and the material rheology. The amount of ink deposited is controlled by the pressure acting in the syringe. Unlike screen-printing, dispenser printing does not require a screen for each layer and the printed pattern can be quickly changed in the computer without the need for additional tooling, allowing one off prototypes. Dispenser printing allows materials with a wider range of viscosities to be deposited than either screen (i.e. $1 \sim 10$ Pa.s [6]) or inkjet (i.e. 0.01 0.04 Pa.s [7]) printing.

This paper reports dispenser printing a proximity sensor on a standard wearable $65 \% / 35 \%$ blend polyester cotton. Such a proximity sensor can be integrated with other printed electronic display devices on fabric to form an interactive textile. The proximity sensor consists of a two-layer structure to detect proximity capacitively. The first layer is an interface layer which smoothes the fabric, the second is the conductor layer which is used to form the sensing plate. The detection distance is determined by the area of the sensing plate as well as the associated electronics.

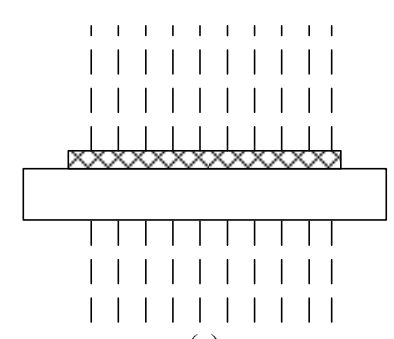

(a)

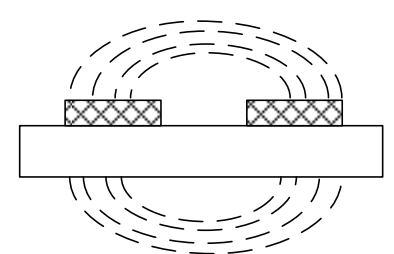

(b)
Figure 1: Two configurations for sensing proximity: (a) single electrode and (b) two in-plane electrodes. 


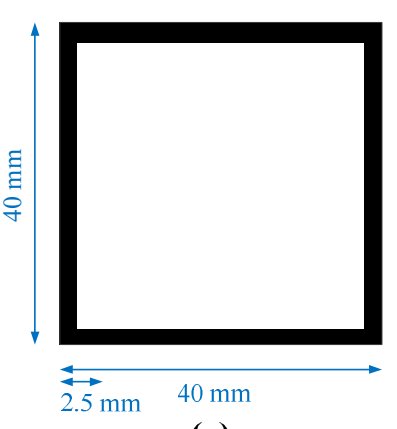

(a)

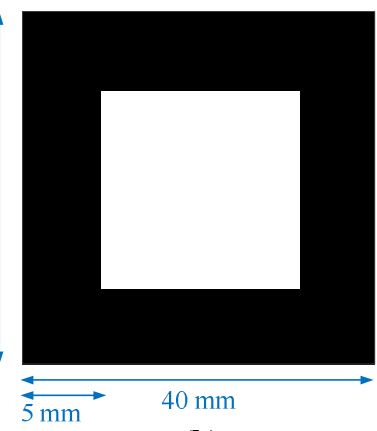

(b)
Figure 2: Proposed proximity sensor design on fabric: (left) $40 \mathrm{~mm}$ by 40 $\mathrm{mm}$ loop; (right) corresponding interface layer.

\section{FABRICATION PROCESS}

\section{A. Proximity sensor design}

There are two potential configurations [8], shown in Figure 1 , of the proximity sensor. Design (a) only requires a single metal plate to form one electrode of the capacitor. The disadvantage is the capacitance could be interfered with by other surrounding objects on the fabric resulting in a false reading. Design (b) consists of two metal plates adjacent to each other on the same plane. One acts as the positive electrode and the other one acts as the negative electrode. The field between the two electrodes is influenced by approaching objects, such as a human hand. The disadvantage of this design is a relatively short detection distance compared to design (a). Therefore, design (a) is selected because it has a simpler structure for printing and offers a longer detectable distance suitable for larger scale installations.

Loop-based proximity sensors, shown in Figure 2, are favourable since the approach reduces conductive ink usage and reduces the area of the fabric being printed so that the printing speed required to produce a large area can be minimised and the impact on the fundamental fabric properties, such as breathability and flexibility, are minimized.

\section{B. Materials and substrate selection}

Two materials, provided by Smart Fabric Inks Ltd. [9], are

TABLE 1: PRINTING PARAMETERS AND CURING CONDITIONS OF THE TWO INKS.

\begin{tabular}{cll}
\hline & \multicolumn{1}{c}{ Interface } & \multicolumn{1}{c}{ Conductor } \\
\hline $\begin{array}{c}\text { Dispensing time } \\
\text { Dispensing pressure }\end{array}$ & $50 \mathrm{~ms}$ & $30 \mathrm{~ms}$ \\
Vacuum pressure & $0.5 \mathrm{kPa}$ & $50 \mathrm{kPa}$ \\
x-resolution & $0.8 \mathrm{~mm}$ & $1 \mathrm{kPa}$ \\
y-resolution & $0.4 \mathrm{~mm}$ & $0.5 \mathrm{~mm}$ \\
Curing & $7.7 \mathrm{~J} / \mathrm{cm}^{2}$ & $0.4 \mathrm{~mm}$ \\
\hline
\end{tabular}

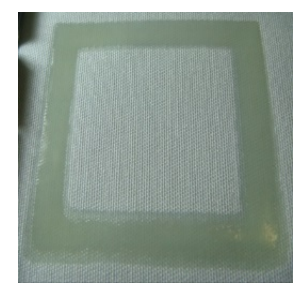

(a)

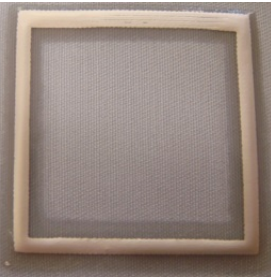

(b)

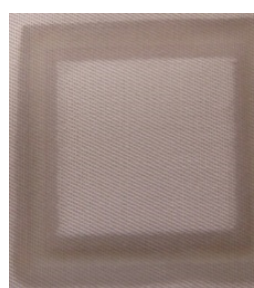

(c)
Figure 3: Printing results on polyester/cotton fabric: (a) interface layer; (b) silver layer; (c) back side of the fabric.

required for the fabrication of the proximity sensor. The first is an insulating interface ink (Fabinks IF-UV-1004) which reduces the roughness of the fabric and the second is a conductor ink (Fabinks TC-C4001) which provides the desired conductivity of the sensing plate. Polyester/cotton $(65 \% / 35 \%)$ fabric is used as the substrate material since it is a widespread fabric used for clothing. Using this fabric provides an example of a typical woven fabric that could be printed; alternative woven fabrics can also be printed using this technique.

\section{Dispenser printing}

3 cc syringes were used to dispense both the conductor and interface inks and the printing parameters are shown in Table 1.

\section{MEASUREMENT PROCRDUE AND RESULTS}

\section{A. Printing results}

Figure 3 shows the printing results of each layer for the proximity sensor on polyester/cotton fabric.

The interface layer achieves a smooth platform for printing the conductor and the printed conductor is smooth and well defined.

\section{B. Proximity sensing circuit design}

To sense the capacitance change in the presence of an approaching object the Microchip MTCH101 proximity sensor IC is used, shown in Figure 4. The maximum detectable distance is defined by the size of the sensor (e.g. $15 \mathrm{~cm}$ range with a $7 \mathrm{~cm}$ circular sensing area) and can be adjusted by varying the potential at the MTSA pin so it can be adapted to different applications [10]. Since Arduino microcontrollers are often already used for smart fabric development, it was chosen for this demonstrator to allow a proximity sensing event to

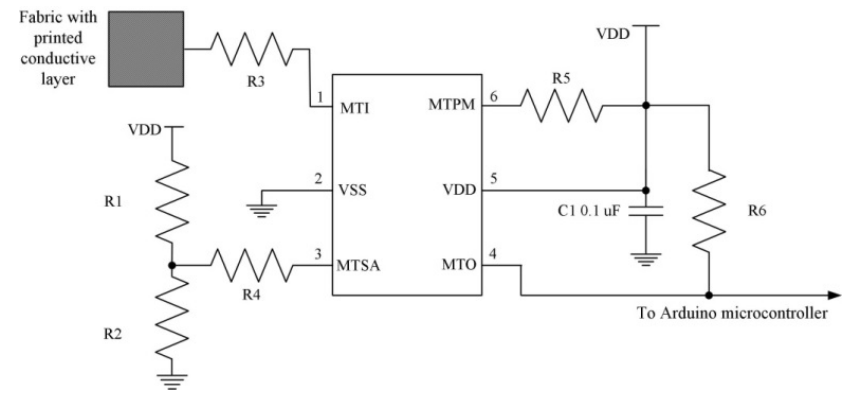

Figure 4: Circuit configuration using microchip proximity sensor IC. 


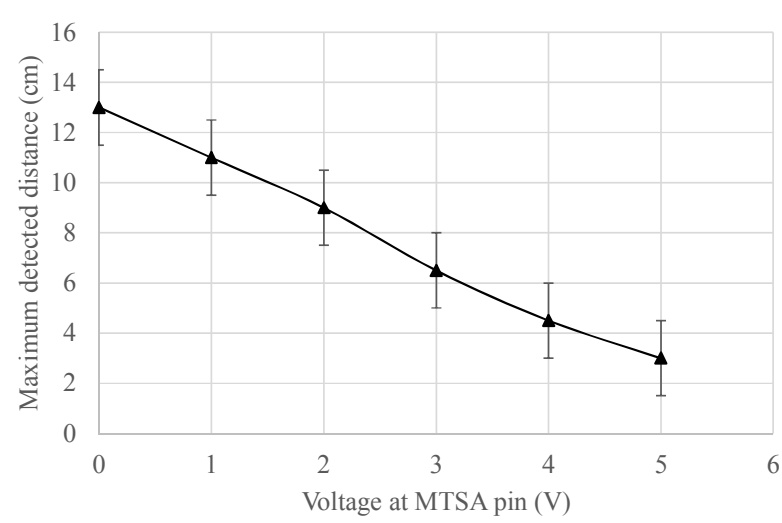

Figure 5: Maximum detected distance of the printed proximity sensor on polyester/cotton fabric.

control an LED. The Arduino was powered by a PC via a USB port. A ruler was placed alongside the sensing plate to measure the vertical detection distance.

\section{Detection results}

To test the entire sensing system, a human hand gradually approached the sensing plate. The maximum detected distance was recorded when the sensor was triggered. Figure 5 shows the maximum detected distance using the printed proximity sensor.

It is shown that, by increasing the potential at the MTSA pin, the maximum detection distance is reduced up to a maximum potential defined by the power supply (i.e. $5 \mathrm{~V}$ ). The detected distance increases linearly with a sensitivity of $2 \mathrm{~cm} / \mathrm{V}$ with a maximum detection distance of approximately $13 \mathrm{~cm}$.

The capacitance of the proximity sensor at each detected distance was measured using a Wayne Kerr 6500b impedance analyser at $1 \mathrm{kHz}$, and is shown in Figure 6.

It is shown that the capacitance of the sensor reduces exponentially as the detected object is moved away from the sensing plate. The change in capacitance is smaller once the distance is above $6 \mathrm{~cm}$. However, it is difficult to obtain a

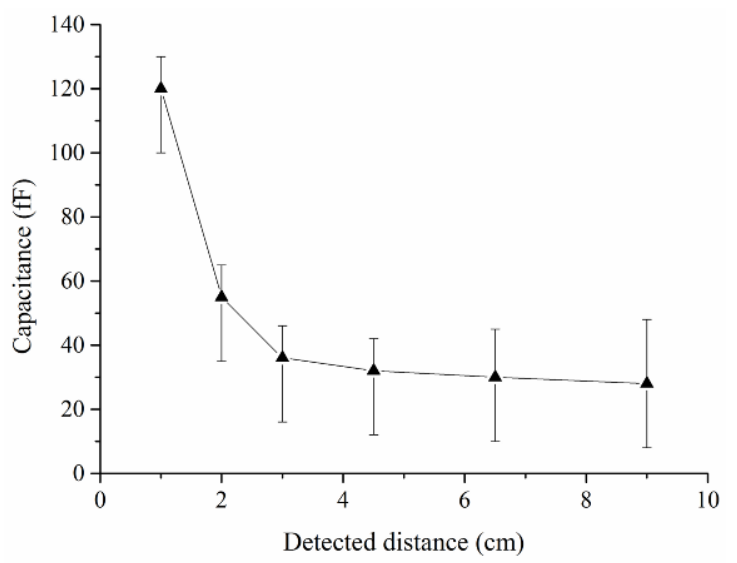

Figure 6: Capacitance as a function of detected distance.

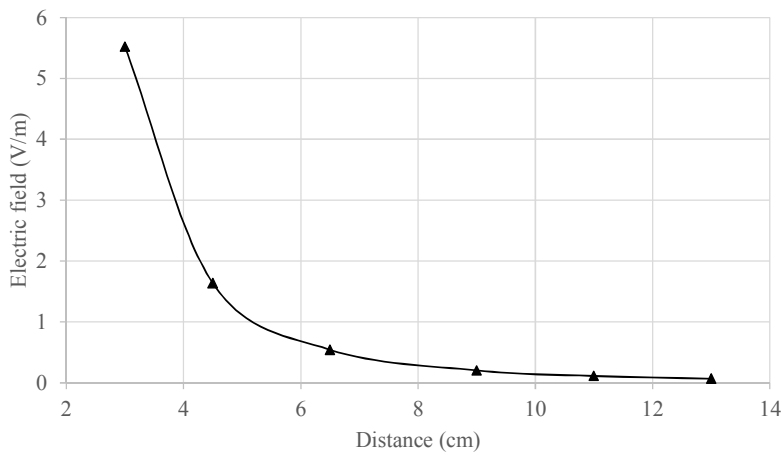

Figure 7: Electric field as a function of the distance.

stable reading at $13 \mathrm{~cm}$

The electric field change for the sensor as a function of the detection distance that is shown in Figure 7. As the distance increases (i.e. the object is further away from the sensing plate), the electric field exponentially reduces because of the change in capacitance.

\section{CONCLUSION}

A capacitive proximity sensor has been fully dispenser printed onto a polyester/cotton fabric in a loop design. The printed sensor is able to detect a proximity event in terms of the presence of a hand although any object can be used. The maximum detected distance is adjusted by changing the potential on the MTSA pin of the microcontroller. An adjustable distance enables the sensor to act as either a proximity or touch sensor without changing either the detection circuit or the printed sensor. This solution therefore offers great versatility and allows the detected distance to be altered depending on different applications.

Dispenser printing enables simple design changes directly on the PC without the need for additional tooling. In addition, dispenser printing does not waste material as the material loaded in a syringe can be used for other designs and ink is only printed where required preserving the fabric's inherent properties.

\section{FUTURE WORK}

Since MTCH101 chip can only detect a proximity event at a fixed sampling frequency, an alternative chip will be selected to enable changing the sampling frequency suitable for different applications.

\section{ACKNOWLEDGEMENT}

The authors would like to acknowledge the funding from the EU (Grant No.: CP-FP-INFSO-FP7-610414) for the smart fabrics research project CREATIF (http://www.creatif.ecs.soton.ac.uk) under which this work was carried out. The authors would like to thank Smart Fabric Inks Ltd for supplying the printable interface and conductive pastes. 


\section{REFERENCES}

[1] S. Fericean and R. Droxler, "New noncontacting inductive analog proximity and inductive linear displacement sensors for industrial automation,” IEEE Sensors Journal, vol. 7, pp. 1538-1545, 2007.

[2] L. Burgi, R. Pfeiffer, M. Mucklich, P. Metzler, M. Kiy and C. Winnewisser, "Optical proximity and touch sensors based on monolithically integrated polymer photodiodes and polymer LEDs," Organic Electronics, vol. 7, pp. 114-120, 2006.

[3] S. Min, J. Kim, H. Shin, Y. Yun, C. Lee and M. Lee, "Noncontact respiration rate measurement system using an ultrasonic proximity sensor," IEEE Sensors Journal, vol. 10, pp. 1732-1739, 2010.

[4] H. Lee, S. Chang and E. Yoon, "Dual mode capacitive proximity sensor for robot applications: implementaion of tactile and proximity sensing capacbility on a single polymer platform using shared electrodes," IEEE Sensors Journal, vol. 9, pp. 1748-1755, 2009.

[5] D. Chrisey, "Materials processing: the power of direct writing," Science, vol. 289, pp. 879-881, 2002.

[6] R. Dorey, Ceramic thick film for MEMS and microdevices, Elsevier Inc..

[7] S. Hoath, G. Martin and I. Hutchings, "Effects of fluid viscosity on drop-on-demand ink-jet break-off," in NIP26 and digital fabrication, 2010.

[8] B. Osoinah, "Proximity capacitive sensor technology for touch sensing applications," Freescale semiconductor Inc. Proximity sensing white paper.

[9] “Fabinks Ltd.," [Online]. Available: http://www.fabinks.com.

[10] Microchip, "Single channel proximity detector MTCH101". 